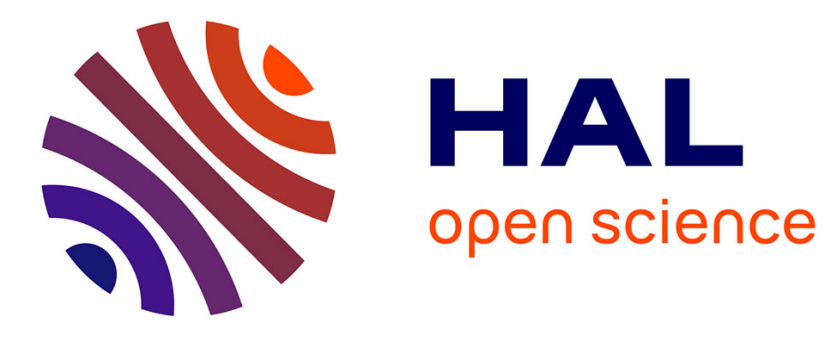

\title{
Energy considerations for a wireless multi-homed environment
}

German Castignani, Nicolas Montavont, Alejandro Martin Lampropulos

\section{To cite this version:}

German Castignani, Nicolas Montavont, Alejandro Martin Lampropulos. Energy considerations for a wireless multi-homed environment. 17th EUNICE Open European Summer School, Energy-Aware Communications - Lecture Notes in Computer Science (LNCS), Sep 2011, Dresden, Germany. pp.177188. hal-00725148

\author{
HAL Id: hal-00725148 \\ https://hal.science/hal-00725148
}

Submitted on 24 Aug 2012

HAL is a multi-disciplinary open access archive for the deposit and dissemination of scientific research documents, whether they are published or not. The documents may come from teaching and research institutions in France or abroad, or from public or private research centers.
L'archive ouverte pluridisciplinaire HAL, est destinée au dépôt et à la diffusion de documents scientifiques de niveau recherche, publiés ou non, émanant des établissements d'enseignement et de recherche français ou étrangers, des laboratoires publics ou privés. 


\title{
Energy Considerations for a Wireless Multi-homed Environment
}

\author{
German Castignani, Nicolas Montavont, and Alejandro Lampropulos
}

Institut TELECOM / TELECOM Bretagne / Université Européenne de Bretagne, 2 rue de la Châtaigneraie, 35576, Cesson Sévigné, France

\{german.castignani, nicolas.montavont,

alejandro. lampropulos\}@telecom-bretagne.eu

\begin{abstract}
Internet wireless access technologies are increasingly heterogeneous. The main cause of this heterogeneity is that, up to now, no wireless technology has succeeded in widely conquering the market. Nowadays, different access technologies co-exist in the same environment allowing multi-homed users to improve their experience by exploiting all available wireless interfaces. However, the energy cost of simultaneously using several wireless interfaces can dramatically drain out mobile devices batteries. In this paper, we review the major work on wireless interfaces energy efficiency (i.e., WLAN and 3G) by highlighting the differences of the proposed energy models and the hardware power consumption. In addition, we present some energy consumption measurements for different application flows and discuss the integration of energy considerations into a general interface selection algorithm.
\end{abstract}

Keywords: Wireless multi-homing, Energy-aware, Interface Selection.

\section{Introduction}

With the recent deployment of heterogeneous wireless technologies, users can exploit network diversity using several wireless interfaces integrated in a single mobile station (MS) to have a better service coverage, a higher throughput and more reliability. Usually, an MS is equipped with a cellular-based interface (e.g., $3 \mathrm{G}$ ) and an IEEE 802.11 interface (WLAN) and it is very common that in most places users have access to different networks using both interfaces. In the case of WLAN, access points (AP) could be residential, public hot-spots or community network deployments 3 . In this context, which is called a multi-homed environment, a user is able to choose which network to use to exchange its data. Moreover, it could exploit several interfaces at the same time by simultaneously assigning different application flows to different interfaces, depending on some pre-defined criteria.

The main goal while taking multi-homing decisions is to match each application flow with the best available wireless interface. Several techniques exist to solve this interface selection problem. In [10, authors compare different multicriteria decision algorithms for interface selection. The decision is taken considering QoS requirements of the different flows, which are weighted using different

R. Lehnert (Ed.): EUNICE 2011, LNCS 6955, pp. 177-188 2011.

(C) Springer-Verlag Berlin Heidelberg 2011 
techniques, such as SAW (Simple Additive Weighting) or TOPSIS (Technique for Order Preference by Similarity to Ideal Solution). These algorithms produce a single-objective optimization to determine the best interface for each application flow. In [7, authors propose a simple energy-aware interface selection technique, which decides to perform a vertical handover (swap all flows from $3 \mathrm{G}$ to WLAN) only if the cost of switching to WLAN (i.e., activating the interface and performing scanning) plus the cost of transmitting the data through WLAN is lower than the cost of continuing to transmit the data through 3G. This approach considers a fixed amount of energy per unit of transported data, which is too simplistic to actually represent the interfaces energy consumption, as we will show in Section 2 ,

In such a multi-homed scenario there is a performance trade-off, since an energy-aware multi-homing decision should maximize the level of QoS of each application flow, while consuming as little energy as possible. To achieve this, the decision mechanism has to consider some inputs. First, to assure a certain level of QoS, a previous knowledge about the MS environment is needed. The decision mechanism must gather information about the presence and performance of different access networks and eventually, the MS position to optimize the network discovery. In addition, to estimate the amount of energy to be consumed by each application flow, the decision mechanism must know the energy consumption of each interface applied to the traffic pattern of the given application. In this paper, we study these very first inputs for the decision making. First, we survey in Section 2 the different aspects of energy efficiency of wireless interfaces, analyzing the power consumption of different interfaces and presenting some energy models to predict energy consumption. Then, in Section 3, we present some energy measurements on different MS, including the impact of transmitting different application flows through different interfaces (WLAN and 3G). We also evaluate the energy impact of the environment discovery and propose some optimizations to achieve more energy efficient WLAN scanning and GPS location. Finally in Section 4 we conclude the paper.

\section{Energy Consumption of Wireless Interfaces}

\subsection{Empirical Studies}

Energy-efficiency of wireless mobile devices has become an important research issue in the last years. Smart-phones, tablets and other battery-based mobile devices have evolved to integrate more and more functionalities, services and applications that have a direct impact on the energy consumption. Some of the contributors of the increasing energy consumption are the communication modules, since most part of these new functionalities use a wireless interface to access the Internet. Moreover, the growth of energy consumption has not been followed by an increasing battery capacity in the same order of magnitude. This produces a degradation of the mobile device autonomy. One way to increase the battery autonomy is to reduce the power consumption of the different hardware components of the mobile device. Another strategy is to optimize the different 
mechanisms and protocols in order to reduce the time the MS spends in high power consuming states.

In a multi-homing context, a user may want to simultaneously use several interfaces to benefit from being connected to different networks. Some authors have studied the energy impact of data communications using different wireless interfaces. In 7, the author presents an experimental benchmarking of energy consumption of WLAN and 3G interfaces. This experiment consists in downloading and uploading data through a WLAN AP and a 3G network for different load rates and radio link quality. By measuring the percentage of remaining battery level along the time, they found that energy consumption is increased by $18.3 \%$ if both WLAN and 3G interfaces are turned on compared to the case in which only $3 \mathrm{G}$ is turned on. Regarding data communications, both interfaces consume the same amount of energy along the time, but a great difference is found when considering the energy consumed by unit of data (MByte). Using the 3G interface, the MS consumes between $0.176 \%$ and $1.81 \%$ of the battery per MByte, while for WLAN, these values are reduced by two orders of magnitude.

Another empirical study was proposed by Xiao et al. [11. The authors evaluated the energy consumption of watching on-line YouTube videos using $3 \mathrm{G}$ and WLAN. under different strategies: online-viewing, local-viewing (i.e., downloadthen-play) and upload. They perform some tests using a Nokia N95 phone and the Nokia Energy Profiler application ( $\mathrm{NEP}^{1}$ ) to gather the instantaneous power consumption of the phone during the experiment. They found for all strategies that the energy consumed by WLAN is lower than by the $3 \mathrm{G}$ interface, which differs with the results presented in [7.

Balasubramanian et al. 2 propose a measurement study for WLAN, 3G and GSM interfaces using Nokia N95 (running NEP) and HTC Fuze with a hardware power meter. They found that using $3 \mathrm{G}$, the MS spends in average $60 \%$ of the total energy by remaining in a high power state during 12 seconds after finishing a transmission (i.e., tail energy). This result is also observed in [9]. GSM behaves in a similar way but the tail time is smaller than in 3G, up to 6 seconds, giving a reduced tail energy. Moreover, the energy consumed during a GSM transmission is higher than in $3 \mathrm{G}$, since due to GSM low data rates, the transmission time becomes longer. Finally, they find WLAN to consume less energy during data transmission, but they highlight the high cost of performing WLAN scanning, as we will analyze in Section 3.2 .

\subsection{Power States of Wireless Interfaces}

Depending on the communication technology, an MS wanting to send or receive data may switch among different power states. A WLAN interface can operate in Continuously Active Mode (CAM) or Power Saving Mode (PSM) as illustrated in Fig. 1a. In CAM, the MS remains in IDLE state if no data communication is being accomplished. In PSM, the MS can switch to a low-power SLEEP state at any moment. In both cases (CAM or PSM), the MS switches between the two

\footnotetext{
${ }^{1}$ http://www.forum.nokia.com/Library/Tools_and_downloads/Other/

Nokia_Energy_Profiler/Quick_start.xhtml
} 

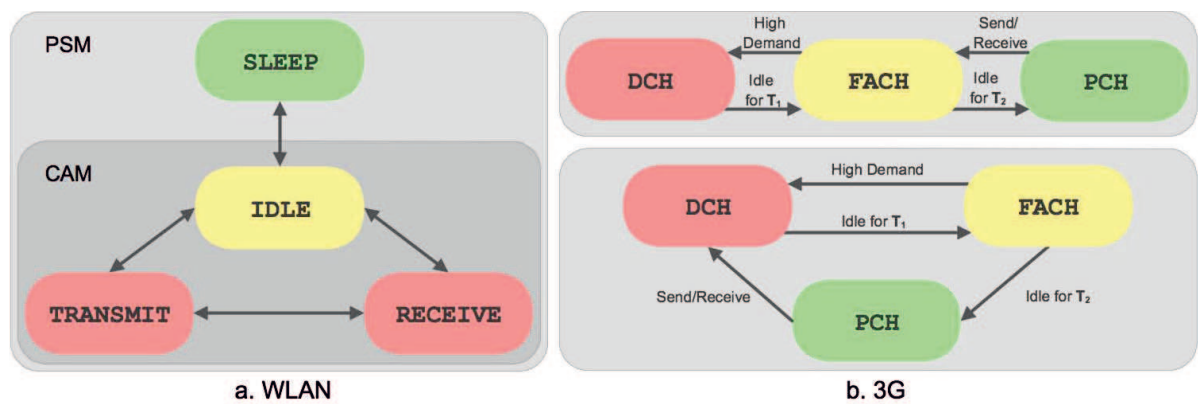

Fig. 1. WLAN and 3G Power States and possible transitions

most power consuming states, RECEIVE and TRANSMIT, when receiving and sending data respectively.

Regarding $3 \mathrm{G}$, transitions between power states are not only related to the amount of data to transmit or receive but to some inactivity timers expiration as well 4. As illustrated in Fig. 1,b, we find three different states depending on the logical channel the MS is using: DCH (Dedicated Channel), FACH (Forward Access Channel) and PCH (Paging Channel). DCH is a high power consuming state that allows sending and receiving data at high data rates. For lower data rates, a common transport channel is used (FACH), involving a lower power consumption. Finally, in the low-power PCH state, no RRC (Radio Resource Control) connection is established, and no data can be transferred (only control data). As it can be seen from Fig. 1,b, transitions between these states can be performed in different manners, depending on the RRC implementation of each network operator. When an MS has some data to transmit or receive, it can directly enter in the DCH state or in the FACH state to switch later to DCH if needed. Since dedicated channels $(\mathrm{DCH})$ are a limited resource, the network operator (through its RRC entity) cannot assign them to an MS for an unlimited time. Moreover, to avoid user performance degradation related to several renegotiations of a $\mathrm{DCH}$, the RRC cannot immediately give the MS access to the FACH. To balance this trade-off, a set of inactivity timers, $T_{1}$ and $T_{2}$ are implemented to manage the transition to lower power states. After being idle for $T_{1}$ in a DCH, the MS can switch to FACH. Then the station can switch again to DCH if the MS demands a high throughput (i.e., higher than a pre-established threshold) or it can switch to PCH after being idle for $T_{2}$. The values of the timers and the state transition policy deeply impact the MS energy consumption and are fixed by the network operator. Then, an MS may consume different amounts of energy depending on the $3 \mathrm{G}$ access network to which it is connected.

Each power state for WLAN or 3G has an associated power consumption. However, these values diverge depending on the specific hardware manufacturer and network operator. In the literature, we can find different power values, as described in Table 1 for WLAN and in Table 2 for 3G. For instance, a WLAN in TRANSMIT state may consume between $1097 \mathrm{~mW}$ and $2000 \mathrm{~mW}$. 
Table 1. WLAN states power consumption ( $\mathrm{mW})$

\begin{tabular}{|l||c|c|c|c|}
\hline WLAN & Nokia N810[12] & HTC G1[12] & Nokia N95[12] & Nokia N90[6] \\
\hline \hline SLEEP & 42 & 68 & 88 & 40 \\
IDLE & 884 & 650 & 1038 & 800 \\
TRANSMIT & 1258 & 1097 & 1687 & 2000 \\
RECEIVE & 1181 & 900 & 1585 & 900 \\
\hline
\end{tabular}

Table 2. WLAN states power consumption (mW)

\begin{tabular}{|l||c|c|c|c|}
\hline 3G & Holma $[\underline{5}$ & Lampropoulos $[\underline{6}$ & Xiao $[11$ & Qian $[\underline{8}]$ \\
\hline \hline PCH & $<18.5$ & 19 & 282 & 0 \\
FACH & $370-740$ & 555 & 549 & $400-460$ \\
DCH & $740-1480$ & 1100 & 742 & $600-600$ \\
\hline
\end{tabular}

\subsection{Energy Models}

While being in operating mode, a wireless interfaces transits among $n$ different power states, $i$, having a pre-defined power consumption, $P_{i}$ (in Watts). In order to predict how much energy an application flow may consume by using a particular interface, one may calculate the amount of time (in seconds) an MS spends in each particular state, $t_{i}$. Then, the energy consumption $C$ (in Joules) can be simply calculated by Eq. 1.

$$
C=\sum_{i=1}^{n} P_{i} \cdot t_{i}
$$

As stated in Section 2, $P_{i}$ mainly depends on the hardware, while $t_{i}$ depends on the particular application flow that has to be transmitted through the interface. Different application flows may need to spend a different amount of time on each particular state. For instance, a web-browsing flow will certainly spend more time in a low-power state (IDLE or PCH) compared to a video-streaming flow. Thus an energy model should first estimate the period of time the MS spends in each state. In the following, we review different energy models found in the literature.

WLAN. In [1, authors propose an energy model for WLAN MSs connected to the same AP for two different kind of TCP traffic: a long file transfer and several short files transfers. In both cases, authors consider two different scenarios: all stations connected to the AP in CAM or all of them in PSM. In the case of a long file transfer authors model the time spent on each operation state by using discrete-time Markov chains. For short files transfer, they focus on the inactivity or think time between downloads and model the system as a Processor Sharing Model, considering exponentially distributed think times and file sizes. Their analytical model corroborates ns- 2 simulations. As expected, for long files, using PSM is less energy-efficient than using CAM because of the overhead of PSPOLL sent to notify the AP that the MS is entering in PSM. On the other 
hand, using PSM for short files, one can triple the number of transferred files per unit of energy.

Another analytical energy model is proposed by Xian et al. 12. In this case, authors generalize the traffic model by considering packet bursts of size $S_{B}$, duration $T_{B}$ and separated by an interval of $T_{I}$. Then, the data rate of the traffic flow is $r=S_{B} /\left(T_{B}+T_{I}\right)$. Their model considers download and upload traffic. In the case of a download, the MS spends some time in IDLE state, some time in the RECEIVE state and, if PSM is activated, the MS can also spend some time in the SLEEP state. Then, the energy consumption can be expressed as $E=P_{R} \cdot T_{B}+P_{I} \cdot T_{\text {timeout }}+P_{S} \cdot T_{\text {sleep }}$, where $T_{\text {sleep }}=T_{I}-T_{\text {timeout }}$ (if $\left.T_{I} \geq T_{\text {timeout }}\right)$. The same rule can be considered for upload traffic, but using $P_{T}$ (transmission power) instead of $P_{R}$ (reception power). Based on the latter expression for $E$, authors calculate, in Eq. 2 the average download power as a function of the data rate $r$. Then, one can use this expression to estimate the average power consumption of a flow on a particular interface by knowing the power level of each state $\left(P_{R}, P_{T}, P_{I}\right.$ and $\left.P_{S}\right)$, the timeout to enter in PSM $\left(T_{\text {timeout }}\right)$ and the characteristics of the flow $\left(T_{I}, T_{B}\right.$ and $\left.S_{B}\right)$.

$$
\begin{aligned}
P_{d}(r)=\frac{E}{T} & =\frac{P_{R} \cdot T_{B}+P_{I} \cdot T_{\text {timeout }}+P_{S} \cdot\left(T_{I}-T_{\text {timeout }}\right)}{T_{B}+T_{I}} \\
& =P_{S}+r\left[\frac{T_{B}}{S_{B}}\left(P_{R}-P_{S}\right)+\frac{T_{\text {timeout }}}{S_{B}}\left(P_{I}-P_{S}\right)\right]
\end{aligned}
$$

Authors instantiate $P_{R}, P_{T}, P_{I}$ and $P_{S}$ for different smartphones (Nokia N810, HTC G1 and Nokia N95) and provide different expressions for the average download $\left(P_{d}(r)\right)$ and upload $\left(P_{u}(r)\right)$ power consumption. They show that analytical results match real measurements on smartphones.

3G. An energy consumption model for $3 \mathrm{G}$ is proposed in [13]. In this model, two different types of traffic are considered: web-browsing (Fig. 2.a) and videostreaming (Fig. 2.b). Web-browsing traffic is modelled as browsing sessions, each one of them composed of packets calls separated by reading intervals. Different browsing sessions are separated by an inter-session time. Video-streaming traffic is modelled as video sessions separated by idle periods. In both cases, the transition between the states (DCH, FACH and $\mathrm{PCH}$ ) is modelled as a discrete-time Markov chain. For web-browsing traffic, they consider that the MS transmits and receives data using a FACH with probability $1-p_{1}$, where $p_{1}$ is the probability that a packet call finishes. If a packet call finishes, the MS remains in the FACH channel until the expiration of the inactivity timer $T_{2}$. The MS can start a new web-session (using a FACH) with probability $p_{2}$, where $p_{2}$ is the probability that the reading time ends. If $T_{2}$ expires, the MS switches to the PCH state and remains there with probability $1-p_{2}$. In the case of video-streaming traffic, the previous Markov chain is extended in order to include the DCH. Then, an MS remains in the $\mathrm{DCH}$ with probability $1-p_{1}^{\prime}$, where $p_{1}^{\prime}$ is the probability that the video session ends. If the video session finishes, $T_{1}$ is triggered and the MS can start a new video session with probability $p_{2}^{\prime}$ at any moment. After $T_{1}$ expiration 


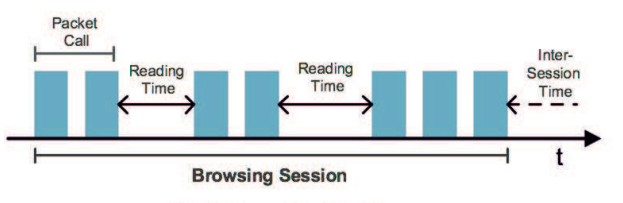

a. Web-browsing Traffic

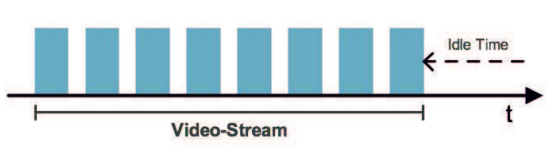

b. Video-Streaming Traffic

Fig. 2. Traffic Modelling

the MS switches to the FACH state and triggers $T_{2}$, and after its expiration, it goes to the $\mathrm{PCH}$ state, remaining there with probability $1-p_{2}^{\prime}$.

Having modelled state transitions as a Markov chain allows obtaining its steady state $(\Pi)$, i.e., the equilibrium distribution of the chain, as a function of $p_{1}$ and $p_{2}$ or $p_{1}^{\prime}$ and $p_{2}^{\prime}$. Then, assuming a fixed traffic session duration, one could calculate the expected energy consumption by multiplying each component of $\Pi$ by its associated power consumption (depending on the interface state, $\mathrm{DCH}, \mathrm{FACH}$ or $\mathrm{PCH}$ ). This allows predicting the amount of energy to be consumed by a particular flow (i.e., different session duration, number of packet calls, reading time, idle time, etc.).

\section{$3 \quad$ Energy Measurements}

\subsection{Energy Cost of Applications Flows}

In order to evaluate the behavior of WLAN and 3G interfaces on energy consumption, we have performed a set of experiments using a multi-interface HTC Dream G1 phone. We have used PowerTutor [14, an Android-based application that allows estimating energy consumption of different smartphone components (wireless interfaces, GPS, audio, CPU and screen). PowerTutor implements an accurate power model that has been derived from an HTC Dream phone that measure energy with at most $2.5 \%$ error. We have calculated the energy consumption of three different applications, web-browsing, YouTube and Skype. Each measurement was performed using only one interface at a time. Regarding Skype, we have calculated the energy consumption for a test call (to a Skype server) and for the application running in background (no active call).

As illustrated in Fig 3 a, we have performed the download of a $45 \mathrm{~s}$ YouTube video using both $3 \mathrm{G}$ and WLAN. We can appreciate that the instantaneous power consumption of WLAN while downloading the video (around $700 \mathrm{~mW}$ ), is higher than the maximum $3 \mathrm{G}$ power consumption (around $570 \mathrm{~mW}$ ) which could produce high energy consumptions for long videos $(22 \mathrm{~J}$ for WLAN against $31 \mathrm{~J}$ for $3 \mathrm{G}$ for $80 \mathrm{~s}$ in our example). The same behavior is observed in a Skype test call (Fig. 3. d), but in this case, the 3G interface remains in a high power consumption state (i.e., the DCH) for around $20 \mathrm{~s}$, before switching to FACH (around $400 \mathrm{~mW}$ ). This gives a higher energy consumption when considering the complete call. Regarding web-browsing (Fig [ b b), we have accessed to the same 

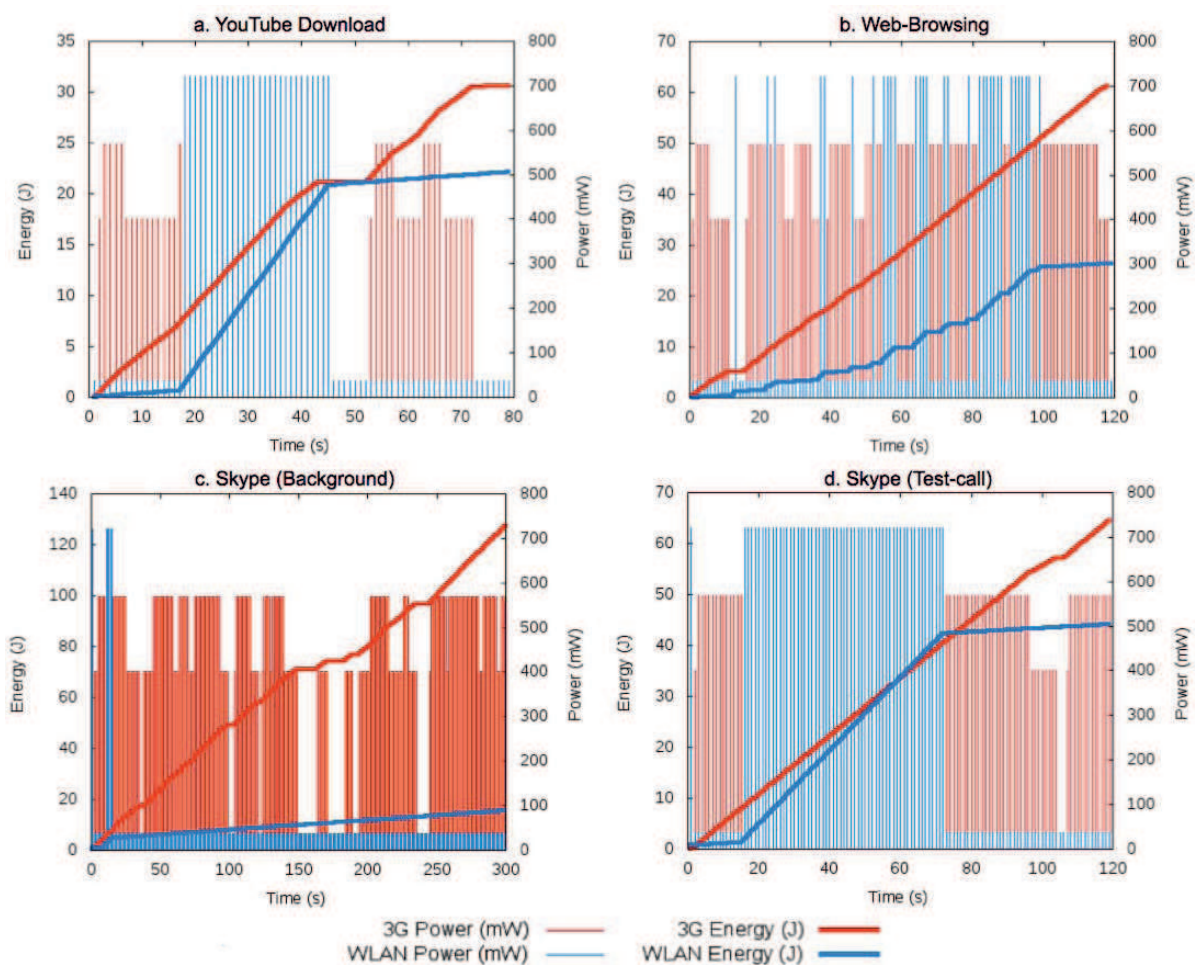

Fig. 3. Energy Measurements using PowerTutor

web-page using both interfaces. Here, we can clearly appreciate the dramatic effect of inactivity timers in $3 \mathrm{G}$, producing a total energy consumption of $62 \mathrm{~J}$ compared to $25 \mathrm{~J}$ for WLAN. The same behavior is found when using Skype in background, with no active calls (Fig 3, c). Here we appreciate that WLAN never enters in its high power state, but $3 \mathrm{G}$ goes into DCH state several times. This issue may be originated by the high amount of data transmitted by the Skype ServiceController module on the background.

We have analyzed the evolution of the power states in both WLAN (Fig. 4.a) and 3G (Fig. 4.b) interfaces during a Skype test call. We observe in these figures that the WLAN states follow the throughput (when the application flow throughput increase or decrease, the WLAN state changes), while in the case of the $3 \mathrm{G}$, we observe that the $\mathrm{DCH}$ is triggered as soon as few bytes are sent over the interface, and the MS stays in this state until the $95^{\text {th }}$ second, i.e., $20 \mathrm{~s}$ after the call was finished.

\subsection{Energy Cost of Scanning and Location Services}

Concerning the interface selection problem, in addition to be able to determine the power consumption for each interface, the MS should acquire an accurate vision of the wireless environment. In order to discover the WLAN available 

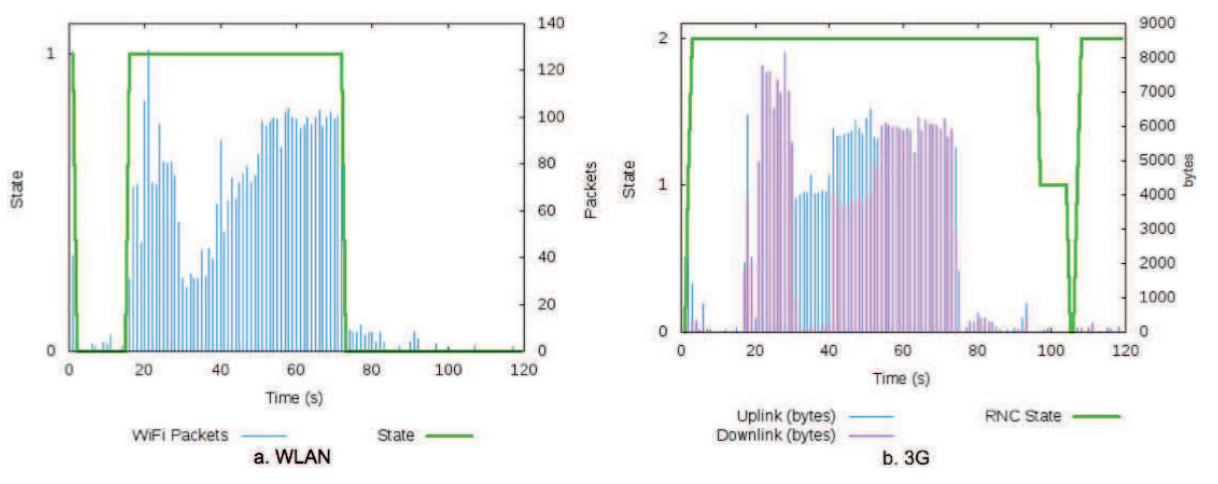

Fig. 4. Skype test call using WLAN and $3 G$ interfaces

networks and their performance, the MS should perform scanning, which requires to actively probe each channel to find out operating APs. Since scanning is an energy-consuming process, we investigate the energy cost of different scanning strategies that use GPS location or sensor-based information. In this experiment, illustrated in Fig. 5 we used a Samsung Galaxy S GT-I9000 with Android OS 2.2 Froyo, and compared the battery drain of the MS in different scenarios (from $100 \%$ to $15 \%$ of the battery capacity). In order to have a baseline, we left the phone idle by turning the screen completely on (red curve) and off (black curve). We observe that having the screen on strongly decreases the battery autonomy by a factor of 13 . With the screen off and without any running application, the battery lasts 30 hours. For all the other tests, the screen was turned off. For the simple case where the MS is continuously scanning every $3 \mathrm{~s}$ (green curve) the battery lasts 11 hours, i.e., three times less than when the device is idle. If for any reason the MS also needs to be located, it has to turn on the GPS and the battery lifetime falls to 9 hours (blue curve). We computed the cost of an individual scanning and found that in average, a single scanning consumes $0.0063 \%$ of the battery, differing from the result presented in [7] which claims $0.122 \%$ of battery drain per scanning for equivalent battery capacities.

Since a typical user does not continuously move, we propose to use sensorbased information to stop scanning when the MS is stationary. By using the internal accelerometer, we detected the MS movement and performed continuous scanning during the following $30 \mathrm{~s}$ from the last detected movement. After this time, if not movement is detected, no scanning process is triggered. In this experiment, the MS is also gathering the GPS location when moving. The different grey curves in Fig. 5 represent the battery drain of such a sensor-aided approach for two different users following different mobility patterns. The first user moved $49.16 \%$ of the time and achieved an autonomy of 11 hours. The second user moved $13.20 \%$ of the time and had an autonomy of 17 hours. We can appreciate that for both mobility patterns we have considered, the energy 


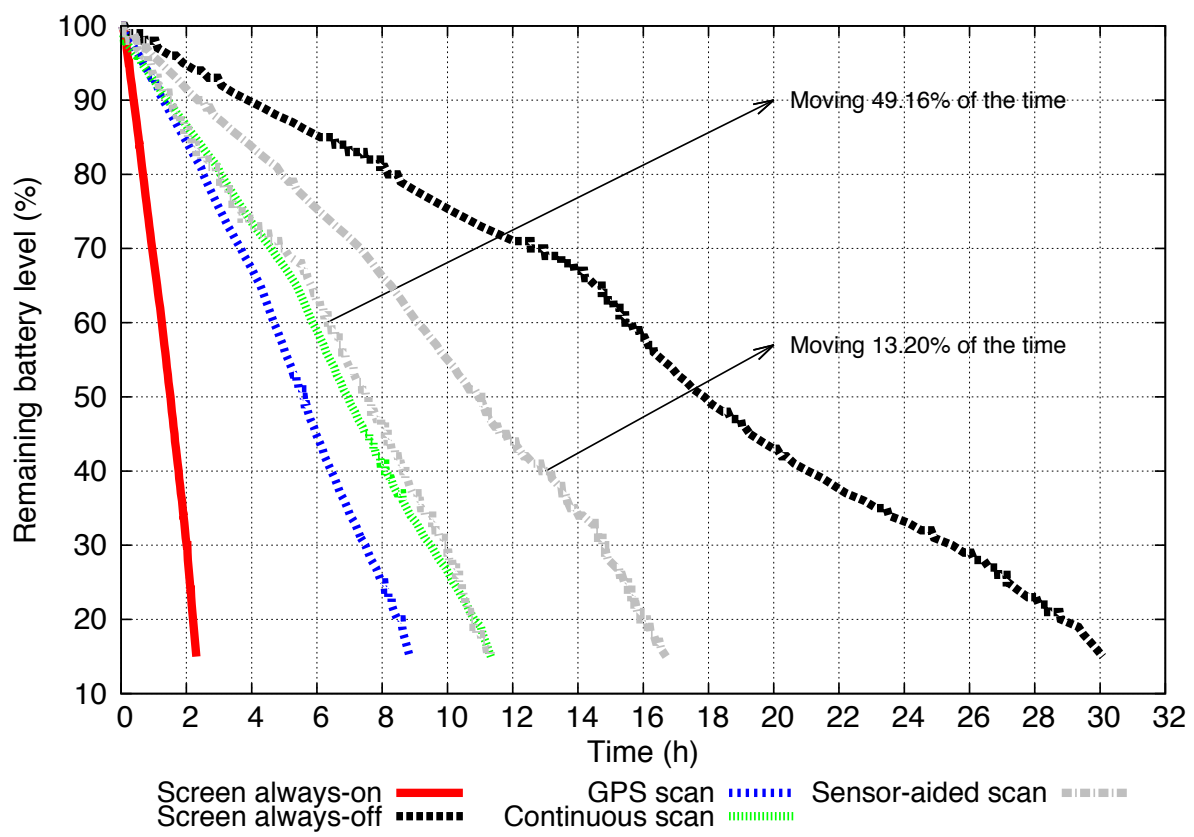

Fig. 5. Scanning and GPS location energy consumption in Samsung Galaxy S

consumed by the sensor-aided strategy is lower than the GPS scan (blue curve), which demonstrates that integrating sensor information while scanning increases the energy efficiency of the MS.

\section{Conclusion and Perspectives}

In this paper, we have reviewed the main findings on energy consumption in a wireless multi-homed environment. In this context, a user wants to improve its experience while communicating through the Internet, but at the same time, he/she wants to maximize the duration of this experience, i.e., the battery autonomy. We showed through energy models and specific measurements that different wireless interfaces consume different amounts of energy for a given application flow. Moreover, for a given wireless technology, different applications (e.g., web-browsing, real-time, interactive) show a different energy consumption. Since multi-homing may potentially involve a high energy-consumption if interfaces are simultaneously used, we need to design schemes that allow intelligent assignation of application flows to the available wireless interfaces. This intelligent assignation can only be achieved by previously knowing the environment, by scanning surrounding networks. We studied the contribution of scanning and GPS location on energy consumption. We found that using smartphone internal sensors can significantly improve energy efficiency depending on the user mobility pattern. 
As future work, we envisage a multi-objective optimization approach to optimize the energy consumption on the one hand and the perceived QoS on the other hand.

\section{References}

1. Agrawal, P., Kumar, A., Kuri, J., Panda, M.K., Navda, V., Ramjee, R., Padmanabhani, V.N.: Analytical models for energy consumption in infrastructure WLAN STAs carrying TCP traffic. In: 2010 Second International Conference on Communication Systems and Networks (COMSNETS), pp. 1-10 (January 2010)

2. Balasubramanian, N., Balasubramanian, A., Venkataramani, A.: Energy consumption in mobile phones: a measurement study and implications for network applications. In: Proceedings of the 9th ACM SIGCOMM Conference on Internet Measurement Conference, IMC 2009, pp. 280-293. ACM, New York (2009)

3. Castignani, G., Loiseau, L., Montavont, N.: An evaluation of IEEE 802.11 community networks deployments. In: 2011 International Conference on Information Networking (ICOIN), pp. 498-503 (2011)

4. Haverinen, H., Siren, J., Eronen, P.: Energy Consumption of Always-On Applications in WCDMA Networks. In: IEEE 65th Vehicular Technology Conference, 2007, VTC 2007-Spring, pp. 964-968 (April 2007)

5. Holma, H., Toskala, A.: HSDPA/HSUPA for UMTS: High Speed Radio Access for Mobile Communications. John Wiley \& Sons, Chichester (2006)

6. Lampropoulos, G., Kaloxylos, A., Passas, N., Merakos, L.: A Power Consumption Analysis of Tight-Coupled WLAN/UMTS Networks. In: IEEE 18th International Symposium on Personal, Indoor and Mobile Radio Communications, PIMRC 2007, pp. 1-5 (September 2007)

7. Petander, H.: Energy-aware network selection using traffic estimation. In: Proceedings of the 1st ACM Workshop on Mobile Internet through Cellular Networks, MICNET 2009, pp. 55-60. ACM, New York (2009)

8. Qian, F., Wang, Z., Gerber, A., Mao, Z.M., Sen, S., Spatscheck, O.: Characterizing radio resource allocation for $3 \mathrm{G}$ networks. In: Proceedings of the 10th Annual Conference on Internet Measurement, IMC 2010, pp. 137-150. ACM, New York (2010)

9. Sharma, A., Navda, V., Ramjee, R., Padmanabhan, V.N., Belding, E.M.: Cooltether: energy efficient on-the-fly wifi hot-spots using mobile phones. In: Proceedings of the 5th international conference on Emerging Networking Experiments and Technologies, CoNEXT 2009, pp. 109-120. ACM, New York (2009)

10. Stevens-Navarro, E., Wong, V.W.S.: Comparison between Vertical Handoff Decision Algorithms for Heterogeneous Wireless Networks. In: IEEE 63rd Vehicular Technology Conference, VTC 2006-Spring, vol. 2, pp. 947-951 (May 2006)

11. Xiao, Y., Kalyanaraman, R.S., Yla-Jaaski, A.: Energy Consumption of Mobile YouTube: Quantitative Measurement and Analysis. In: The Second International Conference on Next Generation Mobile Applications, Services and Technologies, NGMAST 2008, pp. 61-69 (September 2008) 
12. Xiao, Y., Savolainen, P., Karppanen, A., Siekkinen, M., Ylä-Jääski, A.: Practical power modeling of data transmission over $802.11 \mathrm{~g}$ for wireless applications. In: Proceedings of the 1st International Conference on Energy-Efficient Computing and Networking, e-Energy 2010, pp. 75-84. ACM, New York (2010)

13. Yeh, J.-H., Chen, J.-C., Lee, C.-C.: Comparative Analysis of Energy-Saving Techniques in 3GPP and 3GPP2 Systems. IEEE Transactions on Vehicular Technology $58(1), 432-448$ (2009)

14. Zhang, L., Tiwana, B., Qian, Z., Wang, Z., Dick, R.P., Mao, Z.M., Yang, L.: Accurate online power estimation and automatic battery behavior based power model generation for smartphones. In: Proceedings of the Eighth IEEE/ACM/IFIP International Conference on Hardware/Software Codesign and System Synthesis, CODES/ISSS 2010, pp. 105-114. ACM, New York (2010) 